EPJ Web of Conferences 13, 02006 (2011)

DOI: $10.1051 /$ epjconf/20111302006

(c) Owned by the authors, published by EDP Sciences, 2011

\title{
Zero temperature properties of mesons in a vector meson extended linear sigma model
}

\author{
P. Kovács ${ }^{1, a}$, G. Wolf ${ }^{1}$, F. Giacosa ${ }^{2}$, and Denis Parganlija ${ }^{2}$ \\ 1 Research Institute for Particle and Nuclear Physics, H-1525 Budapest, POB 49, Hungary \\ 2 Institute for Theoretical Physics, Johann Wolfgang Goethe University, Max-von-Laue-Str. 1, D-60438 Frankfurt am \\ Main
}

\begin{abstract}
A three flavor linear sigma model with vector and axial-vector mesons is discussed. Preliminary results concerning on the symmetry breaking pattern, the question of parameterization, as well as the resulting meson masses are presented.
\end{abstract}

\section{Introduction}

Effective field theories play a very important role in the investigation of the strong interaction [1], since in the fundamental theory (QCD) lots of questions can not be answered directly due to the complexity of the model. For instance up to now it is still unknown how the mesons and hadrons are built up from the basic degrees of freedom, namely from quarks and gluons. However, in effective field theories, which possesses the same global symmetries (chiral symmetry) as QCD, the meson/hadron spectrum can be investigated thoroughly.

The meson vacuum phenomenology can be analyzed very well in the framework of linear sigma model [2]. In this model the global $U_{L}(3) \times U_{R}(3)$ symmetry of the massless QCD is realized linearly. Since the $U_{L}(3) \times U_{R}(3)$ symmetry is broken due to the axial anomaly [3] to $S U_{A}(3) \times$ $U_{V}(3)$, a $U_{A}(1)$ breaking term is introduced into the Lagrangian of the effective model (see [4] and references therein). The meson fields of the model are placed in $3 \times 3$ matrices (nonets), which transforms according to the adjoint representation of $U_{L}(3) \times U_{R}(3)$. In the present investigation we use an extended version of the linear sigma model, which includes besides the usual scalar and pseudoscalar nonets a vector and an axial-vector nonet as well. thus we are taking into account all the low lying mesonic degrees of freedom.

The experimental data on the majority of mesons are well established [5], however there is still some open questions. For instance the structure of the scalar mesons is still ambiguous [6]. In this paper we present in short our calculation in the extended linear sigma model concerning on the meson spectrum. A more complete analysis will come shortly [7].

The paper is organized as follows, in Sec. 2 the model and the symmetry breaking pattern is presented. In Sec. 3

\footnotetext{
a e-mail: pkovacs@kfki.rmki.hu
}

the tree-level masses are presented with some remarks on the parameterization. We conclude in Sec. 4.

\section{The model}

Our starting point is the $U_{L}(3) \times U_{R}(3)$ symmetric linear sigma model with vector and axial-vector degrees of freedom, and determined by the following Lagrangian

$$
\begin{aligned}
\mathcal{L} & =\operatorname{Tr}\left[\left(D^{\mu} \Phi\right)^{\dagger}\left(D^{\mu} \Phi\right)\right]-m_{0}^{2} \operatorname{Tr}\left(\Phi^{\dagger} \Phi\right)-\lambda_{1}\left[\operatorname{Tr}\left(\Phi^{\dagger} \Phi\right)\right]^{2} \\
& -\lambda_{2} \operatorname{Tr}\left[\left(\Phi^{\dagger} \Phi\right)^{2}\right]+c\left(\operatorname{det} \Phi+\operatorname{det} \Phi^{\dagger}\right)+\operatorname{Tr}\left[H\left(\Phi+\Phi^{\dagger}\right)\right] \\
& -\frac{1}{4} \operatorname{Tr}\left[\left(L^{\mu \nu}\right)^{2}+\left(R^{\mu \nu}\right)^{2}\right]+\frac{m_{1}^{2}}{2} \operatorname{Tr}\left[\left(L^{\mu}\right)^{2}+\left(R^{\mu}\right)^{2}\right] \\
& +\frac{\xi_{1}}{2} \operatorname{Tr}\left(\Phi^{\dagger} \Phi\right) \operatorname{Tr}\left[\left(L^{\mu}\right)^{2}+\left(R^{\mu}\right)^{2}\right]+\xi_{2} \operatorname{Tr}\left[\left(\Phi R^{\mu}\right)^{2}+\left(L^{\mu} \Phi\right)^{2}\right] \\
& +2 \xi_{3} \operatorname{Tr}\left(\Phi R_{\mu} \Phi^{\dagger} L^{\mu}\right)+\mathcal{L}_{3}+\mathcal{L}_{4},
\end{aligned}
$$

where

$$
\begin{aligned}
\Phi & =\sum_{i=0}^{8}\left(\sigma_{i}+i \pi_{i}^{\mu}\right) T_{i}, \\
R^{\mu} & =\sum_{i=0}^{8}\left(\rho_{i}^{\mu}-b_{i}^{\mu}\right) T_{i}, \\
L^{\mu} & =\sum_{i=0}^{8}\left(\rho_{i}^{\mu}+b_{i}^{\mu}\right) T_{i}, \\
H & =\sum_{i=0}^{8} h_{i} T_{i}, \\
D^{\mu} \Phi & =\partial^{\mu} \Phi-i g_{1}\left(L^{\mu} \Phi-\Phi R^{\mu}\right)-i e A^{\mu}\left[T_{3}, \Phi\right], \\
L^{\mu v} & =\partial^{\mu} L^{v}-i e A^{\mu}\left[T_{3}, L^{\nu}\right]-\left\{\partial^{v} L^{\mu}-i e A^{v}\left[T_{3}, L^{\mu}\right]\right\}, \\
R^{\mu \nu} & =\partial^{\mu} R^{v}-i e A^{\mu}\left[T_{3}, R^{\nu}\right]-\left\{\partial^{v} R^{\mu}-i e A^{v}\left[T_{3}, R^{\mu}\right]\right\},
\end{aligned}
$$

This is an Open Access article distributed under the terms of the Creative Commons Attribution-Noncommercial License 3.0, which permits unrestricted use, distribution, and reproduction in any noncommercial medium, provided the original work is properly cited. 
and $T_{i}(i=0 \ldots 8)$ are the generators of $U(3)$. Moreover, $\sigma_{i}$ stands for the scalar, $\pi_{i}$ for the pseudoscalar, $\rho_{i}^{\mu}$ for the vector, and $b_{i}^{\mu}$ for the axial-vector mesons, while $A^{\mu}$ is the electromagnetic field and $h_{i}$ are the constant external fields. $\mathcal{L}_{3}$ and $\mathcal{L}_{4}$ in Eq. (1) contain three and four couplings of the different fields, the explicit forms of which are irrelevant in our present investigation (see e.g. [6]).

In Eq. (1) there is two terms, which breaks the original $U_{L}(3) \times U_{R}(3)$ symmetry, namely the fifth term (the determinant term), and the sixth term (the explicit symmetry breaking term). The first one breaks the $U_{A}(1)$ symmetry, while the second one breaks the complete $U_{A}(3)$ if $h_{0} \neq 0$ and $U_{V}(3) \rightarrow S U_{V}(2) \times U_{V}(1)$ if $h_{8} \neq 0$ (for details see e.g. [4]).

The meson fields $\left(\sigma_{i}, \pi_{i}, \rho_{i}^{\mu}, b_{i}^{\mu}\right)$ do not have well defined quantum numbers that can be obtained with a block diagonal transformation of the form $f_{A}=B_{A i} f_{i}, f \in(\sigma, \pi$, $\left.\rho^{\mu}, b^{\mu}\right)$, where

$$
B=\operatorname{diag}(1, \tau, 1, \tau, \tau, 1), \tau=\frac{1}{\sqrt{2}}\left(\begin{array}{cc}
1 & -i \\
1 & i
\end{array}\right) .
$$

As $A$ goes from 0 to 8 the components of the meson fields goes through on the well known physical particles (for instance in case of the pseudoscalars: $\pi_{0}, \pi^{+}, \pi^{-}, \pi^{0}, K^{+}, K^{-}$, $\left.K^{0}, \bar{K}^{0}, \pi_{8}\right)$, except in the $0-8$ sector, where there is mixing between the particles (in case of the pseudoscalars this means that only a certain linear combination of $\pi_{0}$ and $\pi_{8}$ will be mass eigenstates). For our calculations it is more suitable to choose another base in the $0-8$ sector, which is called the non strange - strange base, and it is given by the following linear transformation,

$$
\begin{aligned}
& f_{N}=\sqrt{2 / 3} f_{0}+\sqrt{1 / 3} f_{8}, \\
& f_{S}=\sqrt{1 / 3} f_{0}-\sqrt{2 / 3} f_{8},
\end{aligned}
$$

where $f \in\left(\sigma, \pi, \rho^{\mu}, b^{\mu}\right)$. To see more explicitly the structure of the $\Phi, L^{\mu}, R^{\mu}$ fields, we give their matrix form (see [8]),

$$
\begin{array}{r}
\Phi=\frac{1}{\sqrt{2}}\left(\begin{array}{ccc}
\frac{\left(\sigma_{N}+a_{0}^{0}\right)+i\left(\pi_{N}+\pi^{0}\right)}{\sqrt{2}} & a_{0}^{+}+i \pi^{+} & K_{S}^{+}+i K^{+} \\
a_{0}^{-}+i \pi^{-} & \frac{\left(\sigma_{N}-a_{0}^{0}\right)+i\left(\pi_{N}-\pi^{0}\right)}{\sqrt{2}} & K_{S}^{0}+i K^{0} \\
K_{S}^{-}+i K^{-} & \bar{K}_{S}^{0}+i \bar{K}^{0} & \sigma_{S}+i \pi_{S}
\end{array}\right), \\
L^{\mu}=\frac{1}{\sqrt{2}}\left(\begin{array}{ccc}
\frac{\rho_{N}+\rho^{0}}{\sqrt{2}}+\frac{a_{1 N}+a_{1}^{0}}{\sqrt{2}} & \rho^{+}+a_{1}^{+} & K^{\star+}+K_{1}^{+} \\
\rho^{-}+a_{1}^{-} & \frac{\rho_{N}-\rho^{0}}{\sqrt{2}}+\frac{a_{1 N}-a_{1}^{0}}{\sqrt{2}} & K^{\star 0}+K_{1}^{0} \\
K^{\star-}+K_{1}^{-} & \bar{K}^{\star 0}+\bar{K}_{1}^{0} & \rho_{S}+a_{1 S}
\end{array}\right)^{\mu},(5) \\
R^{\mu}=\frac{1}{\sqrt{2}}\left(\begin{array}{ccc}
\frac{\rho_{N}+\rho^{0}}{\sqrt{2}}-\frac{a_{1 N}+a_{1}^{0}}{\sqrt{2}} & \rho^{+}-a_{1}^{+} & K^{\star+}-K_{1}^{+} \\
\rho^{-}-a_{1}^{-} & \frac{\rho_{N}-\rho^{0}}{\sqrt{2}}-\frac{a_{1 N}-a_{1}^{0}}{\sqrt{2}} & K^{\star 0}-K_{1}^{0} \\
K^{\star-}-K_{1}^{-} & \bar{K}^{\star 0}-\bar{K}_{1}^{0} & \rho_{S}-a_{1 S}
\end{array}\right)^{\mu} .
\end{array}
$$

The experimentally observed mesons can be assigned to the above fields as follows, $\pi^{ \pm}, \pi^{0}$ and $K^{ \pm}, K^{0}, \bar{K}^{0}$ corresponds to the well-known pion $(\pi(138))$ and kaon $(K(496))$, respectively. The $\pi_{0}, \pi_{8}$ fields are mixture of the $\eta(548)$ and $\eta^{\prime}$ (958) particles. In the scalar sector the assignment is not so obvious, since there are more than one candidate for every scalar fields. In accordance with [6], where the scalar states were found to be above $1 \mathrm{GeV}$, we can assign $K_{S}^{\star \pm}, K_{S}^{\star 0}, \bar{K}_{S}^{\star 0}$ to the $K_{S}^{\star}(1430)$, while $a_{0}^{ \pm}, a_{0}^{0}$ possibly to the $a_{0}(1450)$, respectively. In this sector the mixture of $\sigma_{0}$ and $\sigma_{8}$ can form the $f_{0}(1370)$ and $f_{0}(1710)$ particles. Since this sector is the most uncertain, we would like to use as few of them as it is possible for the parameterization (see Sec. 3), and treat them instead as predictions. The $\rho^{\mu \pm}, \rho^{\mu 0}$ and $K^{\star \pm}, K^{\star 0}, \bar{K}^{\star 0}$ fields represent the $\rho(770)$ and $K^{\star}(892)$ vector mesons, respectively. The remaining two vector meson fields $\rho_{0}^{\mu}$ and $\rho_{8}^{\mu}$ are the mixture of the $\Phi(1020)$ and $\omega(782)$ particles. Finally, the axial-vector meson fields $a_{1}^{\mu \pm}, a_{1}^{\mu 0}$ and $K_{1}^{\mu \pm}, K_{1}^{\mu 0}, \bar{K}_{1}^{\mu 0}$ correspond to the $a_{1}(1260)$ and $K_{1}(1270)$, respectively, while $a_{1,0}^{\mu}$ and $a_{1,8}^{\mu}$ are mixture of $f_{1}(1285)$ and $f_{1}(1420)$.

\subsection{Symmetry breaking}

In this model the chiral symmetry is broken explicitly (the sixth term of Eq. (1)) as well as spontaneously. In case of spontaneous symmetry breaking the effective potential has its minimum at a non-vanishing value, which corresponds to a non-zero expectation value for some of the fields. Since the vacuum has zero quantum numbers the possible fields are the $\sigma_{0}$ and $\sigma_{8}$ scalar fields [4]. Let us denote the expectation values for $\sigma_{0}$ and $\sigma_{8}$ as $\Phi_{0}$ and $\Phi_{8}$, respectively. However, as in case of the fields, it is more convenient to use the non strange - strange base (see Eq. (4)).

According to the usual process, we shift $\sigma_{N}$ and $\sigma_{S}$ by their vacuum expectation values $\Phi_{N}$ and $\Phi_{s}$ and substitute into the Lagrangian. This will result in a technical difficulty, namely that mixing terms appear among certain fields in the Lagrangian.

\section{Tree-level masses}

In order to calculate the tree-level masses after the introduction of the shifts, all the quadratic terms must be considered, which can be written as,

$$
\begin{aligned}
\mathcal{L}^{\text {quad }} & =-\frac{1}{2} \sigma_{A}\left(\delta_{A B} \partial^{2}+\left(m_{\sigma}^{2}\right)_{A B}\right) \sigma_{B} \\
& -\frac{1}{2} \pi_{A}\left(\delta_{A B} \partial^{2}+\left(m_{\pi}^{2}\right)_{A B}\right) \pi_{B} \\
& -\frac{1}{2} \rho_{A \mu}\left(\left(-g^{\mu v} \partial^{2}+\partial^{\mu} \partial^{v}\right) \delta_{A B}-g^{\mu v}\left(m_{\rho}^{2}\right)_{A B}\right) \rho_{B v} \\
& -\frac{1}{2} b_{A \mu}\left(\left(-g^{\mu v} \partial^{2}+\partial^{\mu} \partial^{v}\right) \delta_{A B}-g^{\mu v}\left(m_{b}^{2}\right)_{A B}\right) b_{B v} \\
& -\frac{1}{2} \rho_{A \mu}\left(i g_{1} f_{A B C} \Phi_{C} \partial^{\mu}\right) \sigma_{B}-\frac{1}{2} \sigma_{A}\left(i g_{1} f_{A B C} \Phi_{C} \partial^{v}\right) \rho_{B v} \\
& +\frac{1}{2} b_{A \mu}\left(g_{1} d_{A B C} \Phi_{C} \partial^{\mu}\right) \pi_{B}-\frac{1}{2} \pi_{A}\left(g_{1} d_{A B C} \Phi_{C} \partial^{v}\right) b_{B v},
\end{aligned}
$$

where 


$$
\begin{aligned}
& \left(m_{\sigma}^{2}\right)_{A B}=m_{0}^{2} \delta_{A B}-6 G_{A B C} \Phi_{C}+4 F_{A B C D} \Phi_{C} \Phi_{D} \\
& \left(m_{\pi}^{2}\right)_{A B}=m_{0}^{2} \delta_{A B}+6 G_{A B C} \Phi_{C}+4 H_{A B, C D} \Phi_{C} \Phi_{D} \\
& \left(m_{\rho}^{2}\right)_{A B}=m_{1}^{2} \delta_{A B}+g_{1}^{2} f_{A C M} f_{B D M} \Phi_{C} \Phi_{D}+2 J_{A B, C D} \Phi_{C} \Phi_{D}
\end{aligned}
$$

$\left(m_{b}^{2}\right)_{A B}=m_{1}^{2} \delta_{A B}+g_{1}^{2} d_{A C M} d_{B D M} \Phi_{C} \Phi_{D}+2 J_{A B, C D}^{\prime} \Phi_{C} \Phi_{D}$.

Here $\Phi_{A}$ denotes the vector $\left(\Phi_{N}, 0,0,0,0,0,0,0, \Phi_{S}\right)$, while $f_{A B C}$ and $d_{A B C}$ are the antisymmetric and symmetric group structure constants transformed by (3), viz. $f_{A B C}=$ $f_{a b c} B_{a A}^{-1} B_{b B}^{-1} B_{c C}^{-1}$, and $d_{A B C}=d_{a b c} B_{a A}^{-1} B_{b B}^{-1} B_{c C}^{-1}$. The $G_{A B C}$, $F_{A B C D}, H_{A B, C D}, J_{A B, C D}$, and $J_{A B, C D}^{\prime}$ coefficient tensors contain only the group structure constants and the coupling constants of the Lagrangian. The first two coefficient tensors $G$ and $F$ are totally symmetric, while $H, J$, and $J^{\prime}$ are symmetric in the first two and in the second two indices.

The last four terms of Eq. (6) are mixing terms between different types of mesons. There are two-two terms for the vector-scalar, and for the axial-vector-pseudoscalar mixing. Using the explicit forms of $f_{A B C}$ and $d_{A B C}$ the following mixings are present,

$$
\begin{aligned}
& \pi_{N}-a_{1 N}^{\mu}:-g_{1} \phi_{N} a_{1 N}^{\mu} \partial_{\mu} \pi_{N}, \\
& \pi-a_{1}^{\mu}:-g_{1} \phi_{N}\left(a_{1}^{\mu+} \partial_{\mu} \pi^{-}+a_{1}^{\mu 0} \partial_{\mu} \pi^{0}\right)+\text { h.c., } \\
& \pi_{S}-a_{1 S}^{\mu}:-\sqrt{2} g_{1} \phi_{S} a_{1 S}^{\mu} \partial_{\mu} \pi_{S}, \\
& K_{S}-K_{\mu}^{\star}: \frac{i g_{1}}{2}\left(\sqrt{2} \phi_{S}-\phi_{N}\right)\left(\bar{K}_{\mu}^{\star 0} \partial^{\mu} K_{S}^{0}+K_{\mu}^{\star-} \partial^{\mu} K_{S}^{+}\right)+\text {h.c. } \\
& K-K_{1}^{\mu}:-\frac{g_{1}}{2}\left(\phi_{N}+\sqrt{2} \phi_{S}\right)\left(K_{1}^{\mu 0} \partial_{\mu} \bar{K}^{0}+K_{1}^{\mu+} \partial_{\mu} K^{-}\right)+\text {h.c.. }
\end{aligned}
$$

These mixings can be resolved by appropriate transformations for the $K^{\star \mu}$ vector and the $a_{1}^{\mu}, a_{1 S / N}^{\mu}$, and $K_{1}^{\mu}$ axialvector meson fields. The necessary transformations are the following,

$$
\begin{aligned}
& a_{1 N / S}^{\mu} \longrightarrow a_{1 N / S}^{\mu}+w_{a_{1 N / S}} \partial^{\mu} \pi_{N / S}, \\
& a_{1}^{\mu \pm, 0} \longrightarrow a_{1}^{\mu \pm, 0}+w_{a_{1}} \partial^{\mu} \pi^{ \pm, 0}, \\
& K_{1}^{\mu \pm, 0} \longrightarrow K_{1}^{\mu \pm, 0}+w_{K_{1}} \partial^{\mu} K^{ \pm, 0}, \\
& \bar{K}_{1}^{\mu 0} \longrightarrow \bar{K}_{1}^{\mu 0}+w_{K_{1}} \partial^{\mu} \bar{K}^{0}, \\
& K^{\star \mu^{+}} \longrightarrow K^{\star \mu^{+}}+w_{K^{\star}} \partial^{\mu} K_{S}^{+}, \\
& K^{\star \mu^{-}} \longrightarrow K^{\star \mu^{-}}+w_{K^{\star}}^{\star} \partial^{\mu} K_{S}^{-}, \\
& K^{\star \mu} \longrightarrow K^{\star \mu}+w_{K^{\star}} \partial^{\mu} K_{S}^{0}, \\
& \bar{K}^{\star \mu 0} \longrightarrow \bar{K}^{\star \mu 0}+w_{K^{\star}}^{\star} \partial^{\mu} \bar{K}_{S}^{0} .
\end{aligned}
$$

After transforming the fields with (12) in (6), the $w_{A}$ coefficients can be determined by requiring the disappearance of the mixed terms. It is important to note that this transformation leads to the appearance of multiplicative factors in front of the kinetic terms of the $\pi, \pi_{N}, \pi_{S}, K$, and $K_{S}$ fields, in oder words after the transformations they are not canonically normalized anymore. The multiplicative factors are denoted as $Z_{\pi}, Z_{\pi_{N}}, Z_{\pi_{S}}, Z_{K}$ and $Z_{K_{S}}$. These factors are similar that of the wave function renormalization constants, however, they can take larger values than 1 [6] to the contrary of the usual wave function renormalization constant (see e.g. [9]). Thus in order to get the canonical scalar propagator form, these fields must be renormalized. After a straightforward but lengthy calculation, the coefficients are found to be,

$$
\begin{array}{r}
w_{a_{1 N}}=w_{a_{1}}=\frac{g_{1} \phi_{N}}{m_{a_{1}}^{2}}, \\
w_{a_{1 S}}=\frac{\sqrt{2} g_{1} \phi_{S}}{m_{a_{1 S}}^{2}}, \\
w_{K^{\star}}=\frac{i g_{1}\left(\phi_{N}-\sqrt{2} \phi_{S}\right)}{2 m_{K^{\star}}^{2}}, \\
w_{K_{1}}=\frac{g_{1}\left(\phi_{N}+\sqrt{2} \phi_{S}\right)}{2 m_{K_{1}}^{2}},
\end{array}
$$

while the renormalization factors are,

$$
\begin{aligned}
Z_{\pi} & \equiv Z_{\pi_{N}}=\frac{m_{a_{1}}}{\sqrt{m_{a_{1}}^{2}-g_{1}^{2} \phi_{N}^{2}}}, \\
Z_{\pi_{S}} & =\frac{m_{a_{1 S}}}{\sqrt{m_{a_{1 S}}^{2}-2 g_{1}^{2} \phi_{S}^{2}}}, \\
Z_{K} & =\frac{2 m_{K_{1}}}{\sqrt{4 m_{K_{1}}^{2}-g_{1}^{2}\left(\phi_{N}+\sqrt{2} \phi_{S}\right)^{2}}}, \\
Z_{K_{S}} & =\frac{2 m_{K_{\star}}}{\sqrt{4 m_{K_{\star}}^{2}-g_{1}^{2}\left(\phi_{N}-\sqrt{2} \phi_{S}\right)^{2}}} .
\end{aligned}
$$

Using the following notations, $\Lambda_{N} \equiv \lambda_{1}+\lambda_{2} / 2, \Lambda_{N}^{\prime} \equiv \lambda_{1}+$ $3 \lambda_{2} / 2$, and $\Lambda_{S} \equiv \lambda_{1}+\lambda_{2}$, the tree-level pseudoscalar masses are obtained as

$$
\begin{aligned}
& m_{\pi}^{2}=Z_{\pi}^{2}\left[m_{0}^{2}+\Lambda_{N} \Phi_{N}^{2}+\lambda_{1} \Phi_{S}^{2}-\frac{c}{\sqrt{2}} \Phi_{S}\right] \\
& m_{K}^{2}=Z_{K}^{2}\left[m_{0}^{2}+\Lambda_{N} \Phi_{N}^{2}-\frac{\lambda_{2}}{\sqrt{2}} \Phi_{N} \Phi_{S}+\Lambda_{S} \Phi_{S}^{2}-\frac{c}{2} \Phi_{N}\right] \\
& m_{\pi_{N}}^{2}=Z_{\pi}^{2}\left[m_{0}^{2}+\Lambda_{N} \Phi_{N}^{2}+\lambda_{1} \Phi_{S}^{2}+\frac{c}{\sqrt{2}} \Phi_{S}\right] \\
& m_{\pi_{S}}^{2}=Z_{\pi_{S}}^{2}\left[m_{0}^{2}+\lambda_{1} \Phi_{N}^{2}+\Lambda_{S} \Phi_{S}^{2}\right] \\
& m_{\pi_{N S}}^{2}=Z_{\pi} Z_{\pi_{S}} \frac{c}{\sqrt{2}} \Phi_{N}
\end{aligned}
$$

while the scalar masses are,

$$
\begin{aligned}
& m_{a_{0}}^{2}=m_{0}^{2}+\Lambda_{N}^{\prime} \Phi_{N}^{2}+\lambda_{1} \Phi_{S}^{2}+\frac{c}{\sqrt{2}} \Phi_{S} \\
& m_{K_{S}}^{2}=Z_{K_{S}}^{2}\left[m_{0}^{2}+\Lambda_{N} \Phi_{N}^{2}+\frac{\lambda_{2}}{\sqrt{2}} \Phi_{N} \Phi_{S}+\Lambda_{S} \Phi_{S}^{2}+\frac{c}{2} \Phi_{N}\right]
\end{aligned}
$$




$$
\begin{aligned}
m_{\sigma_{N}}^{2} & =m_{0}^{2}+3 \Lambda_{N} \Phi_{N}^{2}+\lambda_{1} \Phi_{S}^{2}-\frac{c}{\sqrt{2}} \Phi_{S} \\
m_{\sigma_{S}}^{2} & =m_{0}^{2}+\lambda_{1} \Phi_{N}^{2}+3 \Lambda_{S} \Phi_{S}^{2} \\
m_{\sigma_{N S}}^{2} & =2 \lambda_{1} \Phi_{N} \Phi_{S}-\frac{c}{\sqrt{2}} \Phi_{N}
\end{aligned}
$$

where the $m_{\pi_{N S}}^{2}$, and $m_{\sigma_{N S}}^{2}$ are mixing terms in the nonstrange-strange sector. These mixings can be removed by orthogonal transformations, and the resulting mass eigenstates are found to be,

$$
\begin{aligned}
m_{f_{0}^{H} / f_{0}^{L}}^{2} & =\frac{1}{2}\left[m_{\sigma_{N}}^{2}+m_{\sigma_{S}}^{2} \pm \sqrt{\left(m_{\sigma_{N}}^{2}-m_{\sigma_{S}}^{2}\right)^{2}+4 m_{\sigma_{N S}}^{2}}\right] \\
m_{\eta^{\prime} / \eta}^{2} & =\frac{1}{2}\left[m_{\pi_{N}}^{2}+m_{\pi_{S}}^{2} \pm \sqrt{\left(m_{\pi_{N}}^{2}-m_{\pi_{S}}^{2}\right)^{2}+4 m_{\pi_{N S}}^{2}}\right]
\end{aligned}
$$

Using the notations $\Xi_{N} \equiv\left(g_{1}^{2} / 2+\xi_{1}+\xi_{2} / 2\right) / 2, \Xi_{S} \equiv\left(g_{1}^{2}+\right.$ $\left.\xi_{1}+\xi_{2}\right) / 2$, the vector masses are found to be,

$$
\begin{aligned}
m_{\rho}^{2} & =m_{1}^{2}+\frac{1}{2}\left(\xi_{1}+\xi_{2}+\xi_{3}\right) \Phi_{N}^{2}+\frac{\xi_{1}}{2} \Phi_{S}^{2} \\
m_{K^{\star}}^{2} & =m_{1}^{2}+\Xi_{N} \Phi_{N}^{2}+\frac{1}{\sqrt{2}} \Phi_{N} \Phi_{S}\left(\xi_{3}-g_{1}^{2}\right)+\Xi_{S} \Phi_{S}^{2} \\
m_{\omega}^{2} & =m_{\rho}^{2} \\
m_{\Phi}^{2} & =m_{1}^{2}+\frac{\xi_{1}}{2} \Phi_{N}^{2}+\left(\frac{\xi_{1}}{2}+\xi_{2}+\xi_{3}\right) \Phi_{S}^{2}
\end{aligned}
$$

and finally the axial-vector meson masses are given by,

$$
\begin{aligned}
& m_{a_{1}}^{2}=m_{1}^{2}+\frac{1}{2}\left(2 g_{1}^{2}+\xi_{1}+\xi_{2}-\xi_{3}\right) \Phi_{N}^{2}+\frac{\xi_{1}}{2} \Phi_{S}^{2} \\
& m_{K_{1}}^{2}=m_{1}^{2}+\Xi_{N} \Phi_{N}^{2}-\frac{1}{\sqrt{2}} \Phi_{N} \Phi_{S}\left(\xi_{3}-g_{1}^{2}\right)+\Xi_{S} \Phi_{S}^{2} \\
& m_{f_{1}^{L}}^{2}=m_{a_{1}}^{2} \\
& m_{f_{1}^{H}}^{2}=m_{1}^{2}+\frac{\xi_{1}}{2} \Phi_{N}^{2}+\left(2 g_{1}^{2}+\frac{\xi_{1}}{2}+\xi_{2}-\xi_{3}\right) \Phi_{S}^{2}
\end{aligned}
$$

It is worth to note that in case of vectors and axial-vectors there are no mixing terms in the non strange-strange sector.

\subsection{Parameterization}

In order to calculate the tree-level masses in physical units, the unknown parameters of the model must be determined. There are eleven unknown parameters, namely $m_{0}^{2}, m_{1}^{2}, c$, $g_{1}, \lambda_{1}, \lambda_{2}, \xi_{1}, \xi_{2}, \xi_{3}$ and the two condensates $\Phi_{N}, \Phi_{S}$. Since, there are 14 different masses in our model, and all of them are expressed with these parameters, one can choose an appropriate set the - experimentally well established masses, and treat them as a system of equations for the parameters. The system of equations can be solved with multi-parametric minimalization. This work is still ongoing, however some preliminary results can be found in [10].

\section{Conclusion}

We have presented a three flavor linear sigma model with vector and axial-vector degrees of freedom. Implementing the spontaneous symmetry breaking in the model yields not only the known $\pi_{N^{-}} a_{1 N}$ and $\pi^{ \pm, 0}-a_{1}^{ \pm, 0}$ mixings [6] but also the $\pi_{S}-a_{1 S}, K_{S}-K^{\star}$ and $K-K_{1}$ mixings as well. By using the transformations Eq. (12), and subsequently bringing the $\pi_{N, S}, \pi, K_{S}$ and $K$ derivatives to the canonical form, the non-diagonal terms in the Lagrangian can be removed, which leads to the introduction of the pion, kaon, scalar kaon renormalization coefficients. $Z_{\pi}, Z_{\pi_{N}}, Z_{\pi_{S}}, Z_{K}$, and $Z_{K_{S}}$. The tree-level masses than can be expressed with the eleven unknown parameters of the model, which can be determined by using the experimentally well known particle masses [5] and multi-parametric minimalization. Detailed analysis of the different parameterizations and calculations of the decay widths of the resonances in the Lagrangian (1) will be presented in a separate work [7].

\section{References}

1. S. Gasiorowicz, D. A. Geffen, Rev. Mod. Phys. 41, (1969) 531-573

2. M. L evy, Nuovo Cim. 52A, (1967) 23

3. G. 't Hooft, Phys. Rev. Lett. 37, (1976) 8

4. J. T. Lenaghan, D. H. Rischke, and J. SchaffnerBielich, Phys. Rev. D62, (2000) 085008

5. K. Nakamura et al. (Particle Data Group), J. Phys. G 37, (2010) 075021

6. D. Parganlija, F. Giacosa, and D. H. Rischke, Phys. Rev. D82, (2010) 054024

7. D. Parganlija, P. Kovacs, F. Giacosa, Gy. Wolf and D. H. Rischke, in preparation.

8. D. Parganlija, F. Giacosa, D. H. Rischke, P. Kovacs and G. Wolf, To appear in the proceedings of 11th International Workshop on Meson Production, Properties and Interaction (MESON 2010), Cracow, Poland, 10-15 Jun 2010, arXiv:1009.2250 [hep-ph].

9. M. E. Peskin, D. V. Schroeder, An Introduction to Quantum Field Theory (Westview Press, 1995) 211216

10. D. Parganlija, F. Giacosa, P. Kovacs and G. Wolf, arXiv:1011.6104 [hep-ph]. 